\title{
The time required to prepare for a rotated stimulus*
}

\author{
LYNN A. COOPER and ROGER N. SHEPARD \\ Stanford University, Stanford, California 94305
}

\begin{abstract}
Average time required to determine whether an alphanumeric character was presented in its normal version or as its mirror image increased from $500 \mathrm{msec}$ to $1,000 \mathrm{msec}$ as its angular departure from upright increased from 0 to 180 deg. However, when Ss already knew the identity of the upcoming character and when advance information as to its orientation was available for $1,000 \mathrm{msec}$, this reaction time was reduced to about $400 \mathrm{msec}$ regardless of the orientation of the test stimulus. In this case, Ss claimed that they could prepare for the rotated stimulus by imagining the normal version of the designated character rotated into the indicated orientation and that they could then rapidly test for a match against the ensuing stimulus.
\end{abstract}

Shepard and Metzler (1971) have reported that reaction time for determining whether pairs of perspective line drawings depict objects of the same three-dimensional shape increases linearly with the angular difference between the orientations of the two objects. On the basis of the marked linearity of the reaction-time functions as well as the introspective reports of the Ss, Shepard and Metzler argued that the Ss "mentally rotated" a representation of one object of the pair into congruence with the other in order to check for a match or a mismatch.

In two further experiments, Shepard and Klun measured the amount of time required to respond discriminatively to a single rotated stimulus with a natural, preferred orientation (see Shepard, in press). In the first of these further experiments, Ss were presented with alphanumeric characters in various tilted orientations and were required, for each, to determine whether the presented character was the "normal" or the "backward" (i.e., mirror-image) version of that character as generally seen in printed form. Reaction time was found to be a monotonically increasing function of the angular departure of the rotated test stimulus from the standard upright orientation. This finding led Shepard and Klun to suggest that Ss carried out a mental rotation in order to make the "normal"."backward" discrimination. However, this task-unlike the one studied by Shepard and Metzler-required that an internal representation of the visual test character be mentally rotated into congruence

\footnotetext{
*This work was supported by the National Science Foundation (Research Grant GB-31971X) and was completed while the first author was on a National Science Foundation predoctoral fellowship and while the second author was a John Simon Guggenheim fellow at the Center for Advanced Study in the Behavioral Sciences. The authors are indebted to Lincoln Moses for suggestions concerning the statistical analyses, to Ernest Hilgard for the use of a tachistoscope provided by NIMH Grant 03859, and to James Cunningham, Elizabeth Smith, and particularly Christine Feng for various sorts of assistance.
}

with a representation of the normal upright version of that particular alphanumeric character stored in long-term memory.

In both of the experiments mentioned above, the tilted stimulus corresponding to the internal representation that the $\mathrm{S}$ was presumed to be mentally rotating was externally displayed throughout the trial. The question naturally arises as to whether a mental rotation can be carried out in preparation for an ensuing rotated test stimulus. In the second experiment by Shepard and Klun, Ss were again required to identify rotated alphanumeric characters as "normal" or "backward," but this time they were informed in advance as to the identity and the orientation of the test stimulus prior to its actual visual presentation.

When advance information was given (in auditory form) concerning both the identity and the orientation of the ensuing test stimulus (e.g., "the letter $R$ in the $4: 00$ o'clock position"), the function relating reaction time and orientation of the test stimulus flattened considerably and, for some $\mathrm{Ss}$, became virtually horizontal. This finding suggests that Ss were able to prepare for the presentation of the rotated alphanumeric character by using the advance information to generate a mental image of the appropriate character and then to rotate this image into the appropriate orientation. Then, when presented with the actual test stimulus, the $S$ could make the required discriminative response quite rapidly, regardless of the orientation of the test stimulus, by comparing the visual test character against the internally generated and prerotated mental image.

The reaction-time experiment which we report here was designed to explore more fully this process of preparation for the presentation of a rotated visual test stimulus. By manipulating both the orientation of the test stimulus and the duration of advance information concerning that orientation, we sought to obtain more direct evidence for the existence of the hypothesized process of preparatory rotation of a mental image and, especially, to determine the time required to complete such a preparatory rotation. 
ADVANCE INFORMATION TEST
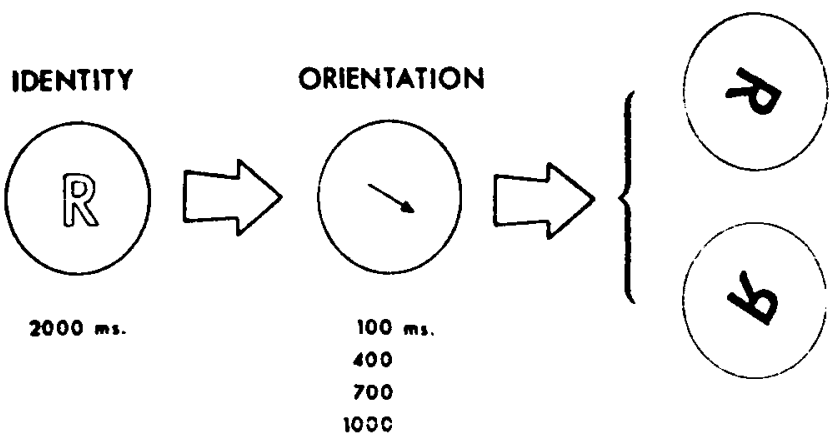

Fig. 1. Sequence of visual displays on a trial of Type B. Each successive display immediately follows the preceding within the same circular field.

\section{METHOD}

\section{Subjects}

The eight Ss, five males and three females, were students and staff at Stanford University.

\section{Stimuli}

The test stimuli were all asymmetrical alphanumeric characters-three uppercase letters $(G, J, R)$ and three Arabic numerals $(2,5,7)$-which could be displayed in either their normal or their backward versions. Each of the six characters could be presented in any of six equally spaced orientations around the circle, in 60-deg steps (with angle of rotation measured as clockwise from the normal upright orientation). The Ss' task was always to discriminate the normal versions of the test characters from the backward or mirror-image versions regardless of their orientation in the picture plane. The appearance of the test character " $R$ " in both the normal and the backward versions at an orientation of $120 \mathrm{deg}$ is illustrated on the right in Fig. 1.

All stimuli, including test characters and advance information cues, were displayed visually within the same circular aperture in a three-field tachistoscope. Figure 1 illustrates the appearance of advance information as to the identity and the orientation of the test character " $R$ " when it was to appear at an orientation of $120 \mathrm{deg}$. Identity information consisted of an outline drawing of the normal upright version of the ensuing test character. Orientation information consisted of an arrow drawn through the center of the circular field and pointing to the position at which the top of the test character was about to appear.

Advance information cues always preceded the test character, and on trials in which both identity and orientation information were provided, the identity cue always preceded the orientation cue. The alphanumeric characters (which served as both identity cues and test stimuli) subtended a visual angle of about $11 / 2 \mathrm{deg}$, and the circular aperture in which all visual displays appeared subtended a visual angle of $4 \mathrm{deg}$. Luminance of all three fields was about $20 \mathrm{fL}$.

\section{Experimental Conditions and General Design}

Each $S$ was tested under eight experimental conditions, differentiated by the type and duration of advance information provided. However, only six of these conditions are relevant for this report-four conditions with different durations of orientation information and two control conditions.

The structure of the four variable-duration conditions is illustrated schematically in Fig. 1. In all four conditions of this type, "B," both identity and orientation information were provided in advance of the actual rotated test character. On all Type B trials, the identity cue was presented for $2,000 \mathrm{msec}$ and was followed immediately by the orientation cue which persisted for $100,400,700$, or $1,000 \mathrm{msec}$, depending upon which of the four Type $B$ conditions was in effect. The onset of the actual test character then coincided with the offset of the orientation cue.

In addition to the four Type B conditions, two control conditions are relevant here. In Condition $\mathrm{N}$, no advance information was provided, only a 2,000-msec blank warning field which preceded the onset of the actual test character. In Condition $\mathrm{C}$, both identity and orientation information were presented in a combined form. The advance information display in this condition consisted of the outline of the normal version of the upcoming test stimulus already rotated to the orientation in which the test character was to appear. This single advance information stimulus was presented for $2,000 \mathrm{msec}$, followed by a 1,000 -msec blank field preceding the onset of the actual test character.

The Ss were instructed to respond to each test stimulus as rapidly as possible without making errors. "Normal" responses were signaled by pressing a right-hand button and "backward" responses by pressing a left-hand button. (However, this response assignment was reversed for the one left-handed $S$.) Trials on which errors were made were later retaken in order to obtain an errorless reaction time for each combination of character, orientation, version (normal or backward), and experimental condition. (This procedure did not significantly increase the length of the experiment, as error rates were low, ranging from $3.6 \%$ to $8.7 \%$ for individual Ss.)

A within-S design was used such that each of the eight Ss saw each of the six characters in both versions (normal and backward) at each of the six orientations under each of the eight experimental conditions (including the two conditions not considered here). The first four $\mathrm{S}$ s were tested on all trials specified by this complete factorial design, which required 576 trials per $\mathrm{S}$ to obtain one observation for each cell of the design. Following an initial practice session, each of these $S s$ was tested for six 1-h experimental sessions. Trials were blocked by conditions, and within each experimental sessions, 12 trials of each of the eight conditions were used. Order of conditions within a session was counterbalanced over sessions. Trials were assigned randomly within blocks with the constraint that each of the six orientations appeared twice within each block. The second four Ss were tested only on trials specified by a "checkerboard" half-replicate of the complete factorial design. Consequently, following the initial practice session, each of these Ss was tested for three 1-h experimental sessions, thus completing 288 trials.

\section{RESULTS AND DISCUSSION}

Figure 2 presents mean reaction time as a function of orientation of the test stimulus, for each of the six experimental conditions described above. The plotted points are averages taken over the six test characters, the two versions (normal and backward), and the eight Ss. (However, these mean curves are quite representative of corresponding curves plotted separately for each of the eight individual Ss.) Although not separately plotted in Fig. 2, "normal" responses were consistently faster than "backward" responses, by some 50-100 msec, for all test stimulus orientations and all experimental conditions. All of the points plotted in Fig. 2 are independent with the exception of the points for $360 \mathrm{deg}$, which are duplicates of the points for $0 \mathrm{deg}$. 
Fig. 2. Mean reaction time plotted as a function of orientation of the test stimulus for the four variable-duration $B$ conditions and for the two limiting control conditions, $\mathrm{N}$ and $\mathrm{C}$. (The points plotted at $360 \mathrm{deg}$ duplicate the points plotted at $0 \mathrm{deg}$.)

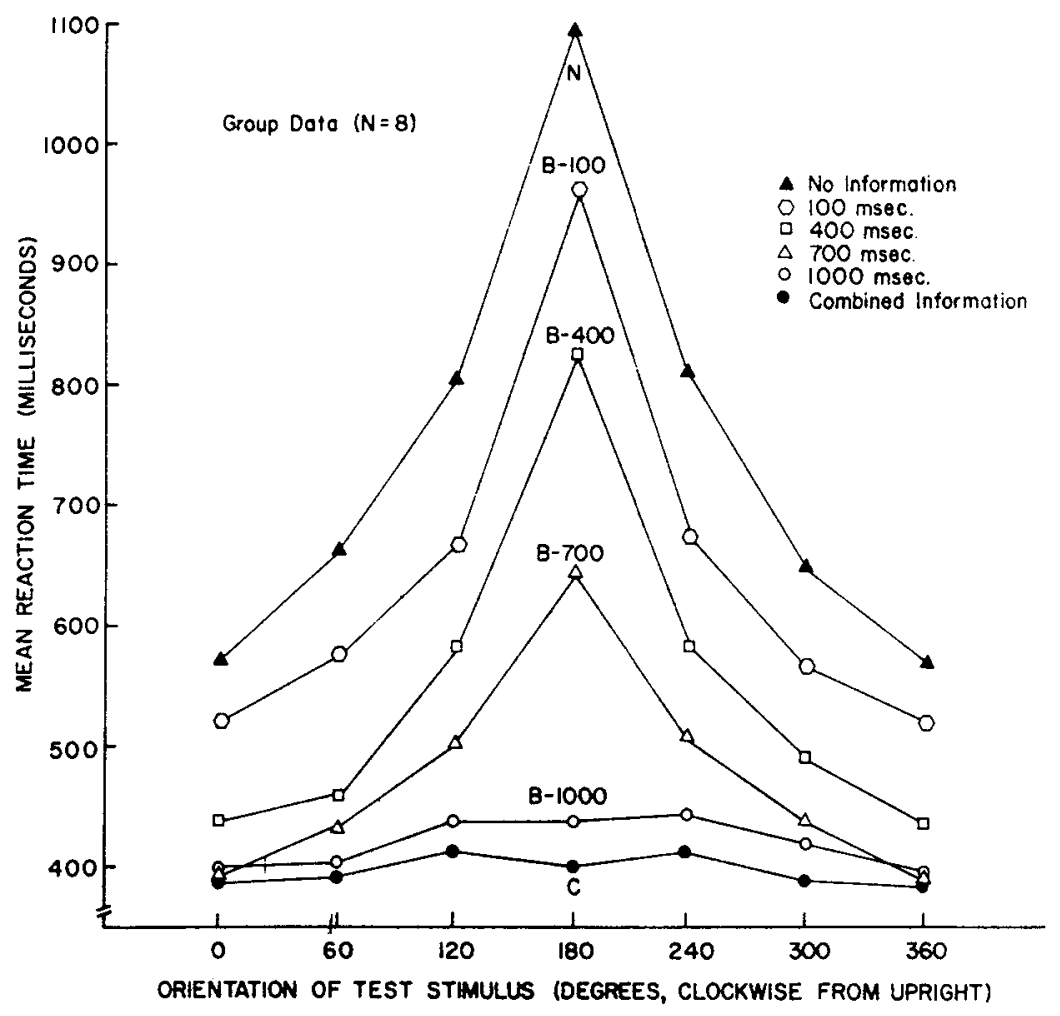

Consider first the uppermost reaction-time function, labeled "N," in Fig. 2. In Condition $N$, no advance information concerning either the identity or the orientation of the upcoming test stimulus was provided. In the absence of advance information, reaction time is a sharply increasing function of angular departure of the test stimulus from the standard upright orientation. Note also the remarkably symmetrical shape of the function about $180 \mathrm{deg}$. This indicates that a given angular departure from upright results in an approximately equal increase in reaction time for both clockwise and counterclockwise rotations.

The marked dependence of reaction time upon departure of the test stimulus from upright, illustrated in Fig. 2, conforms well to the results obtained earlier by Shepard and Klun. Following them and, originally, Shepard and Metzler (1971), we interpret this monotonically increasing function as supportive of the notion that Ss "mentally rotate" an internal representation of the visual test stimulus in order to determine whether that stimulus is normal or backward in version. (In this case, the determination is presumably made by comparing the mentally righted representation of the test character with a representation of the normal version of that alphanumeric character stored in long-term memory.) We attribute the concave-upward monotonic increase in reaction time (rather than linear as in the results of Shepard and Metzler) to the fact that alphanumeric characters (unlike three-dimensional nonsense shapes) have a psychologically unique upright orientation. For a fuller discussion of possible sources of nonlinearity, see Cooper and Shepard (in press).

Consider next the set of reaction-time functions for the Type B conditions, in which information concerning both the identity and the orientation of the upcoming test stimulus was provided in advance of the presentation of the test stimulus itself. The curves labeled "B-100," "B-400," "B-700," and "B-1000" in Fig. 2 show the joint effect of orientation of the test stimulus and duration (in milliseconds) of advance information concerning that orientation upon mean reaction times. When advance information as to the identity of the upcoming test character is presented for $2,000 \mathrm{msec}$, followed by only $100 \mathrm{msec}$ of orientation information (Condition B-100 in Fig. 2), the reaction-time function-though about $100 \mathrm{msec}$ lower-has essentially the same steep shape as that of the function obtained when there was no advance information (Condition $\mathrm{N}$ ). It is evident from Fig. 2, however, that as the duration of the orientation cue is increased (Conditions B-400, B-700, and B-1000), the relationship between reaction time and orientation of the test stimulus becomes weaker. Indeed, when the orientation information is presented for a full $1,000 \mathrm{msec}$, reaction times are short and virtually constant for all orientations of the test stimulus.

An analysis of variance performed on the group data for these four Type B conditions confirms that this progressive flattening of the reaction-time functions is statistically reliable. A four-way analysis of variance (Ss 
by Durations by Orientations by Normal vs Backward Versions) was done with the four-way interaction used as the error term. All main effects were highly significant $(p<.001)$. The sources of variance and the associated Fs were: "Ss," $F=147.25$, df $=7,105$; "durations," $F=$ 266.94, df $=3,105$; "orientations," $F=176.97$, df $=$ 5,105 ; "versions," $F=81.48, \mathrm{df}=1,105$. All two-way interactions with the factor "Ss" were also significant $(\mathrm{p}<.001)$ : "Ss by Durations," $F=13.39, \mathrm{df}=21,105$; "Ss by Orientations," $F=4.56, \mathrm{df}=35,105$; "Ss by Versions," $F=8.05$, df $=7,105$. The only other two-way interaction to achieve statistical significance was that of Durations by Orientations $(\mathrm{F}=18.88$, df $=15,105$, $\mathrm{p}<.001)$. This interaction, of course, corresponds to the flattening of the reaction-time function with increasing duration of orientation information. None of the three-way interactions approached statistical significance. Analyses of variance done on the data of each $S$ individually yielded essentially the same results.

This central finding-that the monotonic increase in reaction time with departure of the test stimulus from upright diminishes, and finally disappears, with increasing advance information duration-dovetails nicely with our notions concerning the process of preparing for a rotated stimulus. If, as we suggested above, Ss prepare for a tilted stimulus by mentally rotating an internal representation of the predesignated character from the upright position into the predesignated orientation, then this process of preparatory mental rotation should take longer to complete as the orientation designated in the advance information departs by larger angles from the upright position.

When this process of preparatory rotation has been fully completed for a given orientation indicated in advance, then reaction time to the actual test stimulus, when presented at that orientation, should be as short as reaction time to a stimulus which has not been rotated. (Presumably, if the $\mathrm{S}$ is fully prepared in the manner described above for the presentation of a rotated character, he can simply compare his prerotated imaginal representation or "mental template" of the designated character with the actual test character in order to determine whether the latter is the normal or backward version.) However, when this preparatory rotation has not been fully completed in advance of the presentation of the test stimulus, then an increase in reaction time to that stimulus should result. For, in that case, additional mental rotation must be carried out after the actual test stimulus has been presented in order for the normal-backward discrimination to be executed successfully.

The flatness of the B-1000 reaction-time function presented in Fig. 2 indicates that this process of preparatory rotation can be completed for all angular departures from upright with a $1,000-\mathrm{msec}$ duration of the orientation cue. When the duration of the orientation cue is reduced to $700 \mathrm{msec}$, the predicted increase in reaction time occurs, especially for test stimuli presented at larger angular departures from upright. With orientation-information durations of 400 and $100 \mathrm{msec}$, very little preparatory rotation can be carried out and reaction times are longer for all test-stimulus orientations.

The reaction-time data presented in Fig. 2 relate well to the introspective reports. The Ss claimed that they did attempt to prepare for the presentation of the test stimulus in these Type B conditions by generating a mental image of the character designated by the identity cue and by then rotating this mental image into the orientation designated by the orientation cue. Most Ss reported that the condition in which $2,000 \mathrm{msec}$ of identity information was followed by $1,000 \mathrm{msec}$ of orientation information provided enough time to rotate a mental image of the anticipated stimulus up to an angular departure of $180 \mathrm{deg}$ from the upright position. In the condition in which the duration of the orientation cue was reduced to $700 \mathrm{msec}$, they claimed that they often had insufficient time to rotate the mental image to $180 \mathrm{deg}$. With further reductions in the duration of the orientation information, rotations of 120 and even $60 \mathrm{deg}$ became difficult. The shortest orientation-information duration (100 msec), Ss claimed, generally allowed for no preparatory rotation before the onset of the actual rotated test stimulus. (The fact that the curve for B-100 nevertheless has a lower overall height than does the curve for $\mathrm{N}$ is to be attributed to the fact that Ss did have some advance information in Condition B-100 and so required less time to process the test stimulus before initiating a rotation. It does not indicate that there was any advance rotation.)

The Ss also said that, under the longer duration conditions, their preparatory mental image of the designated character was sufficiently well defined, even after mental rotation, to be used as a kind of "template" against which to compare the actual visual test stimulus when it appeared. A comparison of the shapes of the B-1000 and C reaction-time functions shown in Fig. 2 lends support to these introspective claims. Recall that in Condition $\mathrm{C}$ the advance information cue consisted of an outline drawing of the normal version of the upcoming character which had already been externally rotated into the appropriate orientation, while in Condition B-1000 the preparatory image had to be generated and mentally rotated by the $\mathrm{S}$ himself. Two analyses failed to indicate any significant difference in the shape of the B-1000 and C reaction-time functions. An analysis of variance indicated that the interaction between "conditions" and "orientations" was nonsignificant $(F<1.0)$, and, when the independent variable was taken as ( 0 to $180 \mathrm{deg}$ ) departure from upright in either direction, a t test for the difference between the slopes of the resulting ("folded") reaction-time functions for Conditions B-1000 and C was also nonsignificant $(t=1.02, d f=7)$. Nonetheless, 
the B-1000 function is slightly above the $\mathrm{C}$ function in overall height by about $20 \mathrm{msec}$ for all orientations, Possibly the preparatory internal representation was slightly more vivid in Condition $\mathrm{C}$ as a result of the fact that complete information as to identity and orientation had been available for $3 \mathrm{sec}$ prior to the onset of the test stimulus in this condition, but for only $1 \mathrm{sec}$ in Condition B-1000.

\section{SUMMARY AND CONCLUSIONS}

When Ss are required to determine whether a tilted alphanumeric character is normal or backward, given no advance information about the test stimulus itself, they mentally rotate an internal representation of the test character, after it has been presented, into the standard upright position and compare this representation with a permanently stored representation of the normal upright version of that character. Evidence for this conclusion derives from the marked increase in reaction time with increasing angular departure of the test character from the upright orientation (Condition $\mathrm{N}$ in Fig. 2).

However, when Ss are given advance information as to the identity and the orientation of the upcoming test character for an adequate amount of time, they can prepare for the presentation of the tilted test stimulus by carrying out a purely mental rotation of a mental image of the anticipated character in advance of its actual presentation. The virtually flat reaction-time function that results when identity information is followed by a full $1,000 \mathrm{msec}$ of orientation information (Condition B-1000 in Pig. 2) provides strong support for this conclusion.

When the duration of the advance orientation information is reduced below the full $1,000 \mathrm{msec}$, this preparatory mental rotation usually cannot be fully completed for large angular departures from upright. In general, the shorter the duration of the advance information, the smaller the angular departure from upright over which preparatory rotation can be completed. The dependence of reaction time upon angular departure of the test stimulus from the upright position for short durations of orientation information and, especially, the weakening of this dependence as the duration of the orientation information increases (Conditions B-100, B-400, and B-700 in Fig. 2) provide support for this conclusion.

Finally, the similarly flat shapes of the reaction-time functions obtained under Conditions B-1000 and C (see Fig. 2) and the introspective reports of the Ss support the following conclusion: For purposes of rapid comparison against the ensuing test stimulus, the internal representation that the $\mathrm{S}$ constructs and rotates on the basis of separately presented cues as to identity and orientation (Condition B-1000) is virtually as effective as an untransformed memory image of the externally rotated character itself (Condition C).

\section{REFERENCES}

Cooper, L. A., \& Shepard, R. N. Chronometric studies of the rotation of mental images. In W. G. Chase (Ed.), Visual information processing. New York: Academic Press, in press.

Shepard, R. N. Studies of the form, formation, and transformation of internal representations. In E. Galanter (Ed.), Cognitive mechanisms. Washington, D.C: Winston \& Sons, in press

Shepard, R. N.. \& Metzler, J. Mental rotation of three-dimensional objects. Science, 1971, 171, 701-703.

(Received for publication November 20, 1972; revision received January 29,1973 .) 